\title{
To Study Levels of LDH in Normal Pregnancy, Pre-Eclampsia \& Eclampsia
}

\author{
Vinaya Vivek Kulkarni ${ }^{1}$, Badrunnisa Shaikh ${ }^{2}$ \\ ${ }^{1}$ Associate Professor, Department of Obstetrics and Gynaecology, Bharati Vidyapeeth (Deemed to be University) \\ Medical College and Hospital, Sangli, Maharashtra, India. ${ }^{2}$ Senior Resident, Department of Obstetrics and Gynaecology, \\ Bharati Vidyapeeth (Deemed to be University) Medical College and Hospital, Sangli, Maharashtra, India.
}

\section{ABSTRACT}

\section{BACKGROUND}

Hypertension during pregnancy is a major health problem. Hypertensive disorders of pregnancy (preeclampsia, eclampsia) are common medical complications and affect $10 \%$ pregnancies. These pregnancies are at increased risk for maternal complications like abruption, disseminated intravascular coagulation, HELLP syndrome, pulmonary oedema, stroke, hepatic failure and renal failure. Foetal complications include growth restriction, prematurity and death of foetus. Early screening of gestational hypertension and preeclampsia may reduce the maternal and foetal complications.

\section{METHODS}

We conducted this study of levels of LDH in normotensive pregnancy, preeclampsia and eclampsia. This is a hospital based comparative cross-sectional study conducted in the department of Obstetrics \& Gynaecology, Bharati Vidyapeeth Medical College and Hospital, Sangli, from December 2015 to July 2017. Out of a total of 150 patients included in present study, 50 patients were in normotensive group, 50 in preeclampsia group and 50 were in eclampsia group. Along with blood investigations, LDH levels were studied in all three groups.

\section{RESULTS}

Mean LDH was 257.24 U/L in normotensive patients as compared to $417.84 \mathrm{U} / \mathrm{L}$ in patients with pre-eclampsia and 565.51 in patients with eclampsia. LDH levels were found to be raised in pre-eclampsia and eclampsia patients and was statistically significant.

\section{CONCLUSIONS}

Serum LDH is the earliest marker in blood during hypoxia and oxidative stress. It is raised in cases of pre-eclampsia and eclampsia. Detection of high-risk patients with increased levels of LDH mandates close monitoring, prompt and correct management to decrease both maternal and foetal morbidity and mortality. Estimation of serum Lactate Dehydrogenase can be used as a prognostic marker for preeclampsia and eclampsia.

\section{KEY WORDS}

Pre-Eclampsia, Eclampsia, Lactate Dehydrogenase (LDH)
Corresponding Author: Dr. Vinaya Vivek Kulkarni, Plot No. 16, Khare Group Housing Society, Dhamani Road, Sangli-416416,

Post-Dhamani, Maharashtra, India.

E-mail: kulkarnivinayav@rediffmail.com

DOI: $10.14260 / j e m d s / 2019 / 600$

Financial or Other Competing Interests: None.

How to Cite This Article: Kulkarni VV, Shaikh B. To study levels of $L D H$ in normal pregnancy, pre-eclampsia \& eclampsia. J. Evolution Med. Dent. Sci. 2019;8(35):2768-2772, 10.14260/jemds/2019/600

Submission 17-06-2019,

Peer Review 16-08-2019,

Acceptance 22-08-2019,

Published 02-09-2019.

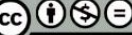




\section{BACKGROUND}

Hypertensive disorder of pregnancy is a common medical complication. It affects $10 \%$ of pregnancies. It accounts for a quarter of all antenatal admissions as it is strongly associated with maternal \& foetal morbidity \& mortality. Gestational hypertension is the new onset of hypertension with blood pressure more than or equal to $140 / 90 \mathrm{~mm}$ of $\mathrm{Hg}$ after twenty weeks of gestation without proteinuria. Pre-eclampsia is gestational hypertension with proteinuria (at least 300 $\mathrm{mg} / 24$ hours).Eclampsia is pre-eclampsia with convulsions. Women with pre-eclampsia are at increased risk for life threatening events, including placental abruption, acute kidney injury, cerebral haemorrhage, hepatic failure or rupture, pulmonary oedema, disseminated intravascular coagulation and progression to eclampsia may progress to eclampsia. Intrauterine foetal growth retardation \& prematurity are common foetal complications. ${ }^{1}$ Early screening of hypertension during pregnancy \& eclampsia may reduce these complications.

Lactate Dehydrogenase (LDH) is an intracellular enzyme which converts pyruvic acid to lactic acid during the process of glycolysis. Hypoxia in preeclampsia enhances glycolysis \& increases LDH activity. Studies have shown that LDH activity and gene expression are high in placentas of pre-eclampsia than normal pregnancy. ${ }^{2-4}$ Hypoxia induces LDH isoenzyme activity in trophoblasts resulting in higher lactate production. Elevated levels of LDH are indicative of cellular damage \& dysfunction. So it can be used as a biochemical marker because it reflects the severity of disease, occurrence of complications \& foetal outcome. Its estimation would prove useful because these complications are preventable. Elevated levels of Lactate Dehydrogenase have also seen in cases of HELLP syndrome. Many authors have used elevated total lactate dehydrogenase (Usually more than $600 \mathrm{U} / \mathrm{L}$ ) as a diagnostic criterion for haemolysis. ${ }^{5-7}$ Among all five isoforms only two of them (LDH 1 AND LDH 2) are released from ruptured red blood cells. ${ }^{8}$ The organ dysfunction in severe pre-eclampsia caused by vascular endothelial damage including maternal liver, kidneys, lungs, nervous system and coagulation system. This will lead to excessive LDH leakage and elevated levels in serum due to cellular dysfunction. The higher levels of serum LDH can be a very useful marker to identify the occurrence of complications of pre-eclampsia in early pregnancy which may reduce the risk of occurrence of disease.

We wanted to find out severity of hypertension in pregnancy with the help of LDH levels and compare the levels of LDH in normotensive pregnancy, pre-eclampsia \& eclampsia.

\section{METHODS}

This is hospital based comparative cross-sectional study conducted in the department of Obstetrics \& Gynaecology, Bharati Vidyapeeth Medical College and Hospital, Sangli, from December 2015 to July 2017. Ethical committee approval taken. All antenatal cases with age ranging from 18 - 35 years \& gestational age ranging from 28 weeks to 40 weeks attending OPD \& admitted under OBGY department of our hospital are included in this study. Consecutive type of nonprobability sampling was followed in present study. 50 pregnant women with preeclampsia, 50 with eclampsia, satisfying eligibility criteria \& given informed consent were included in the study. A total of 50 pregnant women without evidence of hypertension (Normotensive) were taken as controls. Women with any gravidity, age 18-35 years, with informed consent $\&$ hypertension diagnosed first time during pregnancy after 20 weeks were included in study. All pregnant women with chronic hypertension, diabetes, renal disease, liver disease, epilepsy, thyroid disease, megaloblastic anaemia or any medical illness were excluded from study.

A detailed history \& general examination was carried out for all patients. Plain blood sample was taken for analysis of LDH in well-equipped biochemistry laboratory. Complete blood count, Renal function tests, Liver function tests, Bleeding Time, Clotting Time, Serum Uric acid level, viral markers, Blood sugar (Random), Urine routine \& microscopy was also done. Comparison was done between all 3 groups' normotensive, pre-eclampsia \& eclampsia in relation to age, parity, gestational age, presenting symptoms \& S. LDH levels. All the data was entered in Microsoft excel sheet \& then transferred to SPSS Ver. 22 for statistical analysis. Qualitative data was presented as frequency and percentages and analysed using chi-square test while quantitative data was presented as means and SD and analysed using ANOVA test with post-hoc turkey`s test for intergroup comparison.

\section{RESULTS}

Present study included a total of 150 subjects with 50 each of normotensive, pre-eclampsia \& eclampsia. The mean age of the study subjects was 25.16 years with no significant difference between 3 groups. ( $p-0.75)$.

\begin{tabular}{|c|c|c|c|c|}
\hline Group & Number & Mean & SD & p Value \\
\hline Normal & 50 & 25.06 & 3.78 & \multirow{2}{*}{0.75} \\
\cline { 1 - 4 } Pre-eclampsia & 50 & 24.84 & 5.43 & \\
\hline Eclampsia & 50 & 25.58 & 5.61 & \\
\hline \multicolumn{6}{|c|}{ Table 1. Mean Comparison of Age among Study Groups } \\
\hline
\end{tabular}

No significant difference was observed with respect to gravidity, $30.7 \%$ were primigravida while the rest $69.3 \%$ were multigravida.

\begin{tabular}{|c|c|c|c|c|}
\hline \multirow{2}{*}{ Gravidity } & \multicolumn{3}{|c|}{ Group } & \multirow{2}{*}{ Total } \\
\cline { 2 - 5 } & Normal & $\begin{array}{c}\text { Pre- } \\
\text { Eclampsia }\end{array}$ & Eclampsia & \\
\hline \multirow{2}{*}{ Primi } & 13 & 20 & 13 & 46 \\
\cline { 2 - 5 } & $26.0 \%$ & $40.0 \%$ & $26.0 \%$ & $30.7 \%$ \\
\hline \multirow{2}{*}{ Multi } & 37 & 30 & 37 & 104 \\
\cline { 2 - 5 } & $74.0 \%$ & $60.0 \%$ & $74.0 \%$ & $69.3 \%$ \\
\hline p - value 0.21 & \multicolumn{4}{|c|}{} \\
\hline \multicolumn{4}{|l|}{ Table 2. Distribution of Study Groups as Per Gravidity } \\
\hline
\end{tabular}

\begin{tabular}{|c|c|c|c|c|c|}
\hline Variables & Group & N & Mean & SD & p Value \\
\hline \multirow{4}{*}{ LDH levels } & Normal & 50 & 257.24 & 99.61 & \multirow{3}{*}{$<0.01$} \\
\cline { 2 - 5 } & Pre-eclampsia & 50 & 417.84 & 208.58 & \multirow{2}{*}{$<0.51$} \\
\cline { 2 - 5 } & Eclampsia & 50 & 565.51 & 276.72 & \\
\hline
\end{tabular}

Table 3. Mean Comparison of LDH Levels among Study Groups 


\begin{tabular}{|c|c|c|c|c|c|}
\hline \multicolumn{6}{|c|}{ Area Under the Curve } \\
\hline \multirow{2}{*}{$\begin{array}{l}\text { Test Result } \\
\text { Variable(s) }\end{array}$} & \multirow{2}{*}{ Area } & \multirow{2}{*}{ SE } & \multirow{2}{*}{ p Value } & \multicolumn{2}{|c|}{$\begin{array}{c}\text { Asymptotic } 95 \% \\
\text { Confidence Interval }\end{array}$} \\
\hline & & & & $\begin{array}{l}\text { Lower } \\
\text { Bound }\end{array}$ & $\begin{array}{l}\text { Upper } \\
\text { Bound }\end{array}$ \\
\hline $\mathrm{LDH}$ & 0.813 & 0.034 & $<0.01$ & 0.746 & 0.881 \\
\hline \multicolumn{6}{|c|}{ Table 4. Screening Efficacy of LDH Levels to Predict Pre-Eclampsia } \\
\hline \multicolumn{3}{|c|}{ Ideal Cut-Off (Pre- Eclampsia) } & Sensitivity & \multicolumn{2}{|c|}{ Specificity } \\
\hline \multirow{2}{*}{\multicolumn{3}{|c|}{$250 \mathrm{IU} / \mathrm{L}$}} & $83.8 \%$ & \multicolumn{2}{|c|}{$58.0 \%$} \\
\hline & & & $76.8 \%$ & & $0 \%$ \\
\hline \multicolumn{6}{|c|}{$\begin{array}{l}\text { Table 5. LDH Cut-off Level for Pre-Eclampsia and Sensitivity \& } \\
\text { Specificity }\end{array}$} \\
\hline
\end{tabular}

\begin{tabular}{|c|c|c|c|c|c|}
\hline \multicolumn{5}{|c|}{ Area Under the Curve } \\
\hline $\begin{array}{c}\text { Test Result } \\
\text { Variable (s) }\end{array}$ & \multirow{2}{*}{ Area } & SE & $\mathbf{p}$ Value & \multicolumn{2}{|c|}{$\begin{array}{c}\text { Asymptotic 95\% Confidence } \\
\text { Interval }\end{array}$} \\
\cline { 4 - 5 } & & & & Lower Bound & Upper Bound \\
\hline LDH & 0.667 & 0.055 & 0.004 & 0.559 & 0.774 \\
\hline Table 6. Screening Efficacy of LDH Levels to Predict Development of \\
Eclampsia
\end{tabular}

\begin{tabular}{|c|c|c|}
\hline Ideal Cut-Off (Eclampsia) & Sensitivity & Specificity \\
\hline $400 \mathrm{IU} / \mathrm{L}$ & $71.4 \%$ & $44.0 \%$ \\
\hline $450 \mathrm{IU} / \mathrm{L}$ & $63.3 \%$ & $54.0 \%$ \\
\hline Table 7. LDH Level Cut-off for Eclampsia and Sensitivity \& Specificity \\
\hline
\end{tabular}

Common presenting symptoms of pre-eclampsia were pedal edema (42\%) \& epigastric burning (26\%) while that with eclampsia were headache (66\%), pedal edema (56\%) \& epigastric pain (56\%). Mean haemoglobin of normotensive group was significantly higher than preeclampsia group. Coagulation profile was significantly deranged in cases of preeclampsia \& more deranged in eclampsia patients. Mean LDH value was $257.24 \mathrm{U} / \mathrm{L}$ in normotensive patients as compared to $417.84 \mathrm{U} / \mathrm{L}$ in preeclampsia patients \& 565.51 $\mathrm{U} / \mathrm{L}$ in patients with eclampsia. $(\mathrm{p}<0.01)$. It was observed that, LDH levels can significantly predict the development of pre-eclampsia with sensitivity \& specificity of $83.8 \%$ \& $58 \%$ respectively at a cut-off level of $250 \mathrm{U} / \mathrm{L}$ on evaluating the screening efficacy of LDH levels to predict the development of pre-eclampsia via ROC curve. It was also observed that LDH levels can significantly predict development of eclampsia with sensitivity \& specificity of $71.4 \%$ \& $44 \%$ respectively at a cut-off level of $400 \mathrm{U} / \mathrm{L}$ as seen in ROC curve.

\section{DISCUSSION}

Present study was conducted at Bharati Vidyapeeth (Deemed to be University) medical college \& Hospital, Sangli to study the levels of LDH in assessing the severity of hypertensive disorders of pregnancy. The mean age of the study subjects was 25.16 years with no significant difference between the three groups. (p-0.75). Sarkar et al ${ }^{9}$ conducted a study to determine role of serum $\mathrm{LDH}$ as biochemical marker in preeclamptic women and its comparison with normal pregnant women in third trimester. In their study observed the mean age of 23.27 years in normotensive subjects while it was $23.13 \& 23.94$ years in preeclampsia \& eclampsia group respectively. Higher values of serum LDH were found in mild and severe preeclamptic women than those of normal pregnant in third trimester. The study concluded that elevated levels of serum LDH indicates the tissue damage related to endothelial vascular damage, the main cause of the occurrence of preeclampsia. Hak J et al ${ }^{10}$ observed the mean age in controls \& in females with HDP as 25.17 \& 25.75 years respectively $(\mathrm{p}>0.05)$. In another similar study by Kheir et al. ${ }^{11}$ Most women affected by hypertensive disorders of pregnancy with mean age 26.2 years. In our study group, mean age is comparable with above studies.

In present study, no significant difference was observed between the study groups with respect to gravidity. Overall $30.7 \%$ patients were primigravida while rest $69.3 \%$ were multigravidas. In study by Nadkarni et al, ${ }^{12}$ primigravida constituted $49 \%$ of total subjects in study group. Dave et al, ${ }^{13}$ observed half of the cases in their study group as primigravida. Similar results were observed by Kheir et al ${ }^{11}$ where $42 \%$ patients were primigravida. Mean LDH level was $257.24 \mathrm{U} / \mathrm{L}$ in normotensive patients as compared to 417.84 $\mathrm{U} / \mathrm{L}$ in patients with pre-eclampsia \& 565.51 in patients with eclampsia. $(\mathrm{p}<0.01)$. On evaluating the screening efficacy of LDH levels to predict the development of pre-eclampsia \& eclampsia, it was observed that, LDH levels can significantly predict the development of pre-eclampsia \& eclampsia with sensitivity \& specificity of $83.8 \%, 71.4 \%$ \& 58\%, $44 \%$ respectively, at a cut-off level of $250 \mathrm{U} / \mathrm{L} \& 400 \mathrm{U} / \mathrm{L}$.

Preeclampsia is considered an idiopathic multisystem disorder that is specific to human pregnancy. Several biochemical markers have been proposed to predict the severity of pre-eclampsia. ${ }^{14}$. The multi organ dysfunction in preeclampsia caused by vascular endothelial damage, including maternal liver, kidneys, lungs, nervous system, coagulation system will lead to excessive LDH leakage \& elevated levels in serum due to cellular dysfunction. These results are also supported by HS Qublan. ${ }^{15}$ Lactate Dehydrogenase (LDH) is an intracellular enzyme that converts lactic acid to pyruvic acid \& elevated levels indicate cellular death \& leakage of enzyme from the cell.16 The results from our study showed that the levels of serum LDH was significantly higher in pre-eclamptic/ eclamptic women as compared to normal pregnant women is in accordance with the results observed by several other studies. ${ }^{9-13}$ Aziz $\mathrm{R}$ et al, ${ }^{17}$ in a study observed mean serum LDH concentrations significantly higher in pre-eclamptic patients compared to normal pregnant women. (348.34+/- 59.17 vs 255.92+/43.26, $\mathrm{p}<0.01$ ). The study concluded that LDH may be increased due to liver damage. This endothelial vascular damage is the main cause in the occurrence of pre-eclampsia. Higher levels of LDH is very useful marker to identify occurrence of pre-eclampsia. Jyoti Hak et al ${ }^{10}$ conducted a study to correlate LDH levels in normal pregnant women and women with pre-eclampsia and eclampsia in antepartum period and to study the correlation of maternal and foetal outcome with LDH levels. Case control study was done in hundred women with normal blood pressure and hundred women having pre-eclampsia and eclampsia. Serum LDH level was measured using commercially available kits. Similar to our results Hak J. et al. ${ }^{10}$ also observed LDH levels to be gradually increased with the increase in the severity of the disease.

Andrews L et al ${ }^{18}$ conducted a study to assess significance of the value of serum LDH as a marker of Pregnancy Induced Hypertension and its severity. Total 110 cases were studied, 40 were normal pregnant women and remaining 70 were $\mathrm{PIH}$ cases. Out of $70 \mathrm{PIH}$ cases, $15(21.5 \%)$ were mild preeclampsia, $35(50.0 \%)$ were severe pre-eclampsia and 20 $(28.5 \%)$ were eclampsia. Similar to our results, results were observed by Andrews L et al. ${ }^{18}$ with significant rise in the 
LDH levels with increasing severity of the disease, normotensive (172.37 +/- 28.09), mild preeclampsia (356.33+/- 24.47), severe pre-eclampsia (609.91+/- 136.92) \& eclampsia (854.05 +/-247.45) $(\mathrm{p}<0.0001)$. Agrawal $P$ et al. 19 conducted a study to determine the correlation of serum LDH in pre-eclampsia and eclampsia and perinatal outcome. This is a hospital-based case control study done on 140 subjects including normotensive, mild pre-eclamptic, severe pre-eclamptic and eclamptic pregnant women after 28 weeks of gestation before termination of pregnancy. In their study, they observed mean value of serum LDH in control group as $391.4+/-10.9 \mathrm{IU} / \mathrm{L}$, in mild pre-eclampsia as 531.5+/- 24.5 IU/L, in severe preeclampsia as $922.1+/-515.5 \mathrm{IU} / \mathrm{L}$ and in eclampsia 1497.6+/- 602.1 IU/L. The difference in serum LDH level was highly significant $(\mathrm{p}<0.01)$. Afroz et al. ${ }^{20}$ in their cross-sectional study, included 105 pregnant women, 35 were mild preeclamptic, 35 severe preeclamptic and 35 normotensive pregnant women, observed that $82.9 \%$ mild pre-eclamptic and $91.4 \%$ severe pre-eclamptic women had abnormally elevated serum LDH level when a cut-off value of $>200 \mathrm{U} / \mathrm{L}$ was taken. From this study, it can be concluded that elevated serum LDH level was associated with severity of pre-eclampsia. Dave A et al ${ }^{13}$ conducted a study to find out the role of serum Lactate Dehydrogenase in prediction of adverse outcomes of pre-eclampsia and eclampsia i.e. severity of disease and occurrence of complications. A total 200 women were studied; they were divided into control $(n=100)$, severe pre-eclampsia $(n=32)$, eclampsia $(n=68)$. In their study they observed that symptoms and complications of pre-eclampsia along with perinatal mortality were increased significantly in patients with $\mathrm{LDH}>800 \mathrm{IU} / \mathrm{L}$ compared with those who had lower levels.

\section{CONCLUSIONS}

Lactate dehydrogenase is a useful biochemical marker that reflects the severity of pre-eclampsia and can be considered as a prognostic tool from early trimester. Detection of highrisk patients with increased levels of LDH mandates close monitoring, prompt and correct management to prevent maternal and foetal morbidity and mortality. Serum LDH is the earliest marker in blood during hypoxia and oxidative stress. Mean LDH level in our study was $257.24 \mathrm{U} / \mathrm{L}$ in normotensive patients as compared to 417.84 in patients with pre-eclampsia and $565.51 \mathrm{U} / \mathrm{L}$ in patients with eclampsia. Serum LDH is raised in pre-eclampsia and eclampsia. Estimation of serum LDH can be used as a prognostic marker for preeclampsia and eclampsia.

\section{REFERENCES}

[1] Datta D. Hypertensive disorders in pregnancy. In: Konor H, edr. DC Datta`s Textbook of Obstetrics. 7th edn. Kolkata: New Central Book Agency (P) Ltd., 2011: p. 21940.

[2] Tsoi SCM, Zheng J, Xu F, et al. Differential expression of lactate dehydrogenase isoenzymes (LDH) in human placenta with high epression of LDH-A(4) isozyme in the endothelial cells of pre-eclampsia villi. Placenta 2001;22(4):317-22.

[3] Kay HH, Zhu S, Tsoi S. Hypoxia and lactate production in trophoblast cells. Placenta 2007;28(8-9):854-60.

[4] Burd LI, Jones MD Jr, Simmons MA, et al. Placental production and foetal utilization of lactate and pyruvate. Nature 1975;254(5502):710-11.

[5] Tompkins MJ, Thiagarajah S. HELLP (hemolysis, elevated liver enzymes and low platelet count) syndrome: the benefit of corticosteroids. Am J Obstet Gynecol 1999;181(2):304-9.

[6] O`Brien JM, Milligan DA, Barton JR. Impact of high dose corticosteroid therapy for patients with HELLP (Hemolysis, Elevated Liver enzymes and Low Platelets) syndrome. Am J Obstet Gynecol 2000;183(4):921-4.

[7] O`Brien JM, Shumate SA, Satchwell SL, et al. Maternal benefit to corticosteroid therapy in patients with HELLP (Hemolysis, Elevated Liver enzymes and Low Platelets ) syndrome: impact on the rate of regional anesthesia. Am J Obstet Gynecol 2002;186(3):475-9.

[8] Barton JR, Sibai BM. Diagnosis and management of hemolysis, elevated liver enzymes and low platelets syndrome. Clinics in Perinatology 2004;31(4):807-33, vii.

[9] Sarkar PD, Sogani S. Evaluation of serum lactate dehydrogenase and gamma glutamyl transferase in preeclamptic pregnancy and its comparison with normal pregnancy in third trimester. Int J Res Med Sci 2013;1(4):365-8.

[10] Hak J, Nayar-Un-Nisa, Gupta S. LDH levels in pregnancy and its association with severity of the disease and fetomaternal outcome in pre-eclampsia and eclampsia. JK Sciences 2015;17(3):110-15.

[11] Kheir AEM, Ali RBA, Kononna AAM. Neonatal outcome in hypertensive disorders of pregnancy in a tertiary neonatal unit in Sudan. Journal of Medicine and Medical Research 2014;2(5):59-65.

[12] Nadkarni J, Bahl J, Parekh P. Perinatal outcome in pregnancy associated hypertension. Indian Pediatrics 2001;38(2):174-8.

[13] Dave A, Maru L, Jain A. LDH (Lactate Dehydrogenase): a biochemical marker for the prediction of adverse outcomes in preeclampsia and eclampsia. The Journal of Obstetrics and Gynecology of India 2016;66(1):23-9.

[14] Page NM. The endocrinology of pre-eclampsia. Clin Endocrinol (Oxf) 2002;57(4):413-23.

[15] Qublan HS, Ammarin V, Bataineh O, et al. Lactic Dehydrogenase as a biochemical marker of adverse pregnancy outcome in severe pre-eclampsia. Med Sci Monit 2005;11(8):CR393-7.

[16] Krefetz RG. Enzymes. Clinical Chemistry. $4^{\text {th }}$ edn. Philadelphia: Lippincott Williams and Wilkins 2000: p. 196-8.

[17] Aziz R, Mahboob T. Relation between preeclampsia and cardiac enzymes. ARYA Atheroscler 2008;4(1):29-32.

[18] Andrews L, Patel N. Correlation of serum lactate dehydrogenase and pregnancy induced hypertension with its adverse outcomes. International Journal of Research in Medical Sciences 2016;4(5):1347-50. 
[19] Agrawal P, Rajoria L, Vyas J, et al. Serum LDH in preeclampsia and eclampsia and maternal outcomes. Sch J App Med Sci 2016;4(6C):2052-5.
[20] Afroz R, Akhter QS, Sadia H, et al. Serum Lactate Dehydrogenase (LDH) level in severe preeclampsia. Journal of Bangladesh Society of Physiologist 2015;10(2):71-5. 